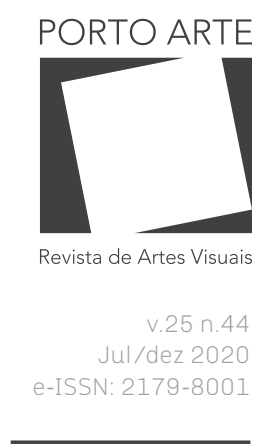

\section{Susana Caló}

ORCID: 0000-0002-2784-1294

Faculty of Arts and Humanities, King's College London, United Kingdom

\section{Resumo}

Este ensaio enceta uma discussão em torno do protocolo experimental chamado 'grille', desenvolvido na clínica La Borde fundada por Jean Oury em 1952. 0 ensaio aborda o projecto da psicoterapia institucional refletido na grade. Oferece uma visão da grade como um instrumento de criação coletiva contínua da instituição de cuidado, com efeitos terapêuticos para todas as pessoas envolvidas, desde o pessoal até aos residentes e monitores. Descrevendo como a grade funcionava através da criação de ligações entre o material e o semiótico, o mental e o organizacional, o social e o ambiental, com o efeito de não só singularizar a trajetória da própria instituição, mas também daqueles que nela vivem, o ensaio salienta a importância dos processos analíticos institucionais em projectos emancipatórios.

Palavras-chave

Análise Institucional, Félix Guattari, Auto-gestão. Clínica La Borde. Grade

\section{Abstract}

This text presents a close discussion of the experimental protocol called 'grid' as developed at the clinic La Borde founded by Jean Oury in 1952. The paper teases out the institutional psychotherapy project reflected in the grid. It offers a view of the grid as an instrument of continuous collective creation of the institution of care, with therapeutic effects to all persons involved, from staff to residents and monitors. Describing how the grid worked by the creation of connections between the material and the semiotic, the mental and the organisational, the social and the environmental, to the effect of not only singularising the trajectory of the institution itself, but also of those living in it, the paper highlights the importance of institutional analytical processes in emancipatory projects.

Key Words

Institutional analysis. Félix Guattari. Self-management. La Borde Clinique. Grid.

\section{A Grade ${ }^{1}$}

The Grid
1- Este texto foi publicado pela primeira vez em inglês como 'The Grid' (2016) Earth, Tecnosphere iss Anthropocene Curriculum \& Campus, House of World Cultures (HKW), realizado em Berlim. Disponível em <https: //www.anthropocenecurriculum.org/contribution/ the-grid>. A presente tradução é de Felipe Drago. 


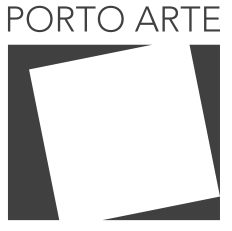

Revista de Artes Visuais

V. $25 \mathrm{n} .44$ Jul/dez 2020 e-ISSN: 2179-8001
Figura 1 e 2: Exemplar de uma grade publicada em "Histoires de La Borde: 10 ans de psychothérapie institutionnelle à la clinique de Cour-Cheverny 1953-1963," Recherches, vol 21. Março-Abril (1976)
"Não vejo contradição entre institucionalização e capacidade criadora." Félix Guattari (1986, p. 120)

"Existe uma velocidade de subjugação que se opõe aos coeficientes de transversalidade" Gilles Deleuze e Félix Guattari (1996, p. 366)
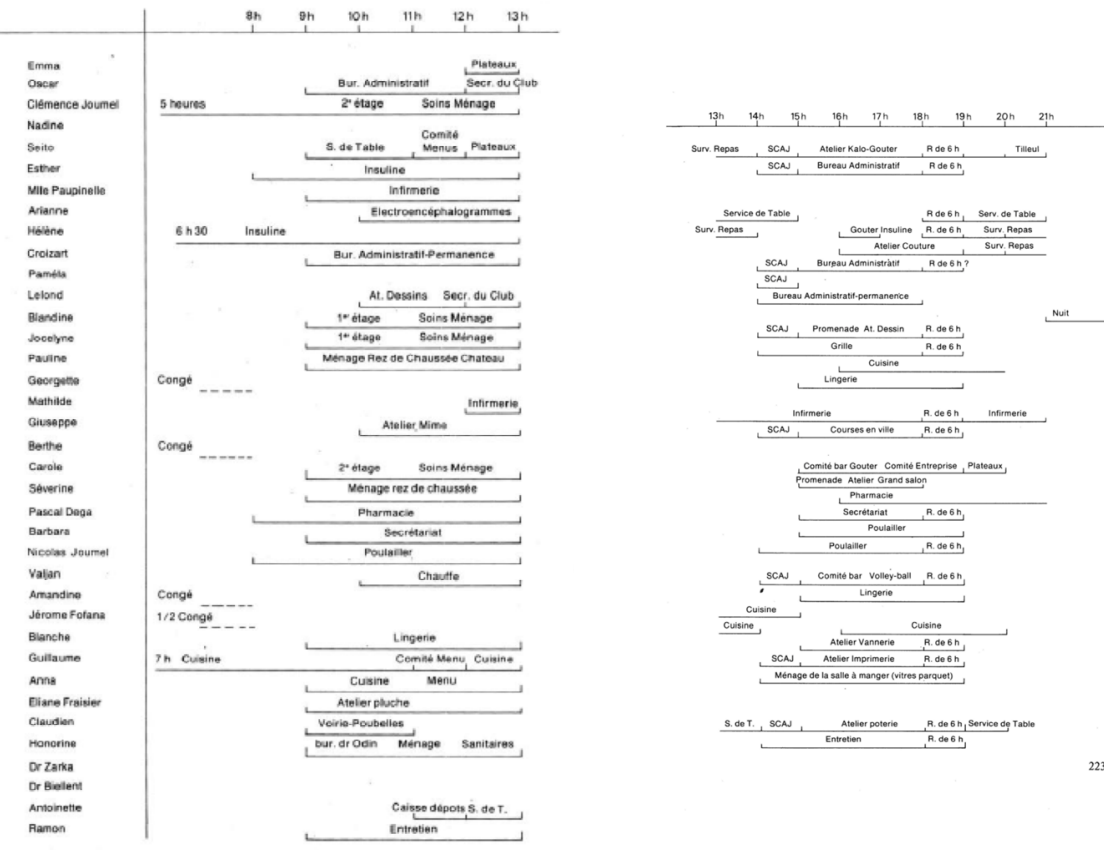

La Borde Clinique ocupa um lugar importante na história da psiquiatria francesa por conta de seu protagonismo no que veio a ser conhecido como movimento da psicoterapia institucional. A clínica foi inaugurada em 1952 por Jean Oury, a quem se juntou logo depois por Félix Guattari ${ }^{1}$. Oury considerava que as instituições psiquiátricas estavam doentes e que era necessário tratá-las para tratar os pacientes. Esse efeito patogênico do meio ambiente foi chamado de "patoplástico" (patoplastique). Daí a famosa expressão: "Tratar os doentes sem tratar o hospital é loucura!" Isso significava considerar o hospital em suas dimensões sociais e políticas.

Uma série de protocolos organizacionais foi estabelecida em La Borde com o objetivo principal de estimular a autonomia dos pacientes, permitindo que eles recuperassem um senso de responsabilidade e "reapropriassem o significado de sua existência

1- A figura fundadora da psicoterapia institucional foi François Tosquelles, psiquiatra catalão que trabaIhava no hospital Saint Alban, durante a Ocupação Alemã na França, e onde ocorreu o primeiro experimento em psicoterapia institucional 


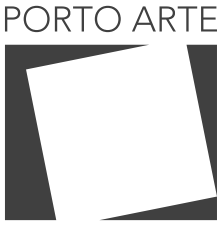

Revista de Artes Visuais

v. 25 ก. 44

$\mathrm{Jul} / \mathrm{dez} 2020$ e-ISSN: 2179-8001

em uma perspectiva ética e não mais tecnocrática" (Guattari, 1995). Uma nova dinâmica espacial também foi implementada para evitar o reforço das estruturas de poder. Para dar alguns exemplos, os pacientes seriam livres para caminhar para onde quisessem e os espaços associados às funções médicas deveriam rotar. Igualmente, considerava-se que medicamentos não deveriam ser administrados sempre pela mesma pessoa. Com essas medidas, o escopo da análise deixaria de ser limitado à privacidade e seria estendido a toda a instituição. Para Oury e Guattari, o tecido da dinâmica da vida diária de La Borde foi pensado para oferecer oportunidades analíticas de diversos tipos.

Para além desses protocolos organizacionais experimentais desenvolvidos em La Borde, um em particular merece nossa atenção - "a grade" - como exemplificação do potencial emancipatório dos processos institucionais.

\section{O protocolo}

A grade era um cronograma de trabalho rotativo, dividido por tarefas e atividades. Ela mostrava o nome das pessoas e a quantidade de tempo por semana que cada uma passaria em cada tarefa ou atividade. Visualmente consistia em dois eixos - um eixo vertical, com uma lista dos nomes das pessoas encarregadas de tarefas ou atividades específicas, e um eixo horizontal de tempo, das $8 \mathrm{~h}$ às $21 \mathrm{~h}$ (Figura 1 ).

Surgindo por volta de 1957, a grade emerge da necessidade de "enquadrar a desregulamentação"2 (palavras de Guattari, 1998), ao mesmo tempo em que tentava preservar a atmosfera amigável da clínica (em seus primeiros anos, La Borde era amplamente autogerenciada e espontaneamente organizada). Um exemplo de grade da década de 1960 inclui na lista de tarefas lavar louça, limpeza, deveres da cozinha e do turno da noite, servir à mesa, além de muitas outras. As atividades eram coisas como os clubes, o jornal ou a lavanderia.

As tarefas e atividades eram distribuídas de acordo com seu grau de agradabilidade3. As tarefas estavam associadas à "desagradabilidade" e as atividades à "agrabilidade". As tarefas, diferente das atividades, deveriam ser compartilhadas por todos para garantir o mínimo de funcionamento diário da clínica e, portanto, eram consideradas responsabilidade de todos. Para cada uma das tarefas havia pontos relativos ao seu valor e relativos ao número de vezes que uma pessoa a executava (frequência). Os pontos eram acumulados e podiam ser usados para barganhar no momento de discutir a grade diária.

Dessa forma, a definição de tarefas e atividades funcionou como um indicador do que a maioria das pessoas que residiam na instituição considerava mais ou menos agradável. Um exemplo disso foi a lavanderia, à qual vários textos se referem como sendo uma tarefa que todos desejavam realizar. Do ponto de vista da análi-

\footnotetext{
2- Ver Félix Guattari, "La Grille'”, Chimères, vol. 34, Autumn (1998): p. 1; também online <https://www.revue-chimeres.fr/Chimeres-no-34-La-grille>. Este é um texto datilografado de uma apresentação feita por Guattari em 1987 na La Borde e posteriormente publicada em Chimères com o mesmo título. 3- Ver "Histoires de La Borde: 10 ans de psychothérapie institutionnelle à la clinique de Cour-Cheverny 1953-1963," Recherches, vol. 21, March-April (1976). Ver também o estudo detalhado acerca de La Borde desenvolvido pelo CERFI: L'Institutionalisation des collectifs de travail. Monographie sur la clinique de La Borde. Paris: Centre d'études, de recherches et de formation institutionnelles, 1974.
} 


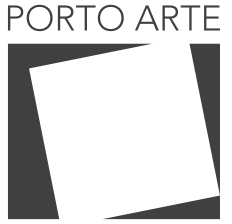

Revista de Artes Visuais

v.25 n. 44 Jul/dez 2020 -ISSN: 2179-8001
Figura 3. Sistema de pontos para tarefas e actividades exemplificado em "Histoires

de La Borde: 10 ans de psychothérapie institutionnelle à la clinique de Cour-Cheverny 1953-1963," |Recherches, vol 21, Março-Abril (1976)
TABLEAU I SIMPLIFIE DES TACHES ET DES ACTIVITES

assumées par le personnel , roulant s pendant une semaine

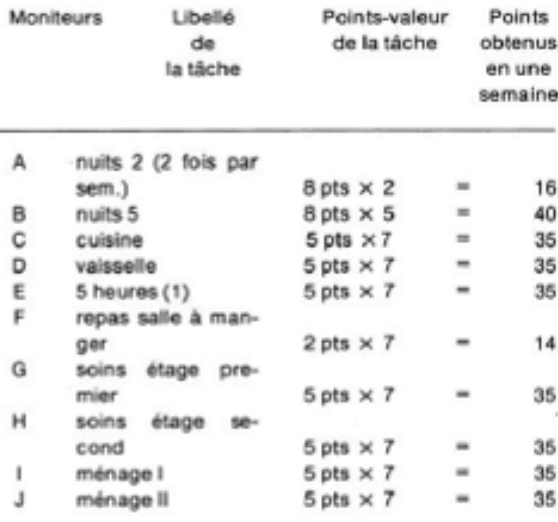

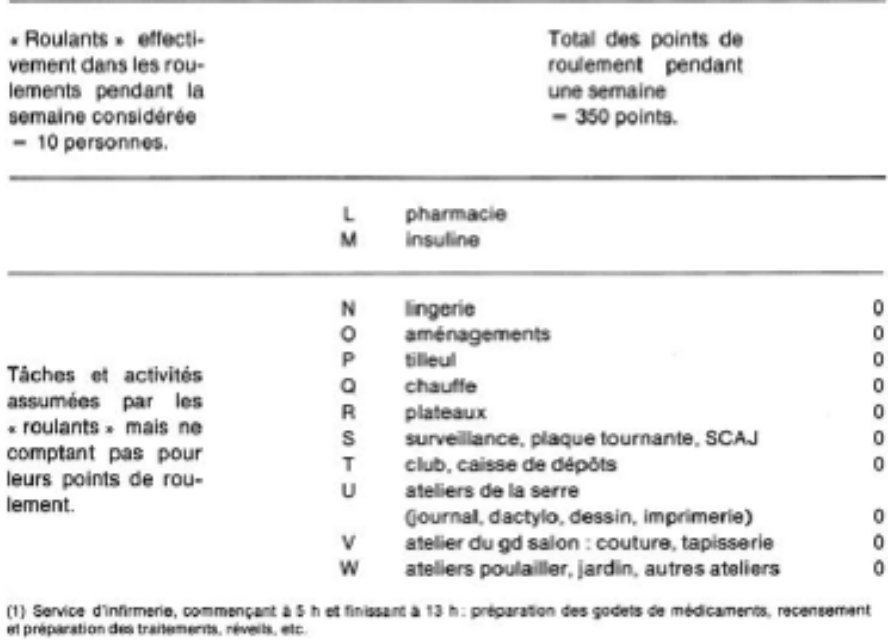

se institucional, esse aspecto aparentemente sem importância abriria uma janela para outra coisa - algo que, de outra forma, passaria despercebido. A grade permitiu traçar mutações do desejo e dos investimentos subjetivos na medida em que foram expressos no nível da dinâmica institucional. Ao mesmo tempo, como protocolo organizacional, tornou visíveis as relações de poder - em particular, no que toca a todos aqueles aspectos deixados de fora da tradicional relação médico-paciente. Também trouxe à tona as relações existentes em segundo plano: o contexto institucional, suas restrições, sua organização, suas práticas específicas e assim por diante. A cada evento institucional, material ou imaterial, discursivo ou não discursivo, foi atribuído um potencial expressivo. ${ }^{4}$

4- Nesse sentido, Guattari se refere a Claude Poncin, que propôs o conceito de "situèmes" para se referir às relações institucionais no contexto de La Borde (em referência à ideia de fonemas na linguagem, as estruturas básicas da linguagem) - "situèmes" constituiria assim a "unidade básica da linguagem" em La Borde. Veja Guattari, "La 'Grille'" (1998) p. 5 


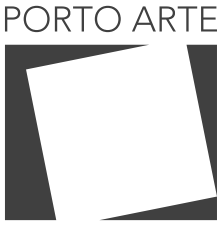

Revista de Artes Visuais

$\vee 25 n .44$

$\mathrm{Jul} / \mathrm{dez} 2020$ e-ISSN: 2179-8001

Nesta visão, a análise institucional é fundamentalmente coletiva. Isso não ocorre apenas porque, em seu significado estrito, a análise não é mais um privilégio apenas do terapeuta, mas é coletivizada - i.e., ocorre coletivamente (em sessões de grupo, discussão, etc.). Antes de tudo, ela é coletiva porque é impactada por disposições espaciais, dimensões linguísticas e significantes, fatores técnicos, econômicos e sociológicos, e não puramente por disposições pessoais e individuais.

A elaboração conceitual guattariana da grade fornece uma visão do que estava em jogo a partir um protocolo tão simples de organização e quantificação. No texto "La 'Grille", Guattari se refere à grade como um "sistema articulatório" cujo objetivo era "tornar articulável a organização do trabalho com as dimensões subjetivas, de modo a permitir que certas coisas saiam à luz do dia, possibilitando a existência de certas superfícies de inscrição" (Guattari, 1998, p. 12). Tome como exemplo a composição da equipe que gerencia a grade (os grilleuses). Essa equipe, ela própria rotativa, não deveria ser composta por médicos para evitar que a divisão do trabalho e a estruturação cotidiana da clínica se congelassem em torno da mesma estrutura médica. A medida não tinha como objetivo negar a perícia médica, mas impor limites à organização do trabalho proveniente de uma única fonte: em outras palavras, permitir que o enquadramento da organização ou estrutura provenha de várias fontes locais, e não de uma fonte sobrejacente.

Não causa surpresa, portanto, enxergar o conflito ao qual a criação da equipe de gerenciamento (grilleuses) ou a criação da grade diária estavam sujeitas ${ }^{5}$. Processos constantes de negociação e discussão coletivas ocorreram. No entanto, o conflito foi vital para expor estruturas dominantes e tornar visíveis as estruturas de poder em jogo.

\section{Linguagem local e semiótica não-redutiva}

Com base nisso, era fundamental que a grade fosse discutida e negociada entre os afetados por ela. Como comenta Guattari, "não adianta jogar alguém de pára-quedas em uma tarefa - principalmente se for estratégico - sem o seu consentimento, sem saber como é para ele naquele momento do dia em relação ao resto do seu tempo de trabalho e, sobretudo, em relação ao que realmente gostaria de estar fazendo" (Guattari, 1998, p. 8).

É evidente que a grade, descrita por Guattari como uma "discursividade analítica coletiva", era muito mais do que um mero esquema rotativo. À medida que a atribuição de tarefas e a rotação eram objeto de feedback e revisão, a própria grade moldou e revelou sistematicamente os processos institucionais que se desenrolaram. Desta forma, através da mudança e constituição da instituição, da mesma forma que em organograma em evolução, a grade foi um instrumento de design institucional coletivo. Em essência, teve a virtude de singularizar a própria trajetória da instituição com aqueles que nela viviam.

\footnotetext{
5- Uma edição especial da Recherches intitulada "La Borde: 10 ans de psychothérapieinstitucionalle à la clinique de Cour-Cheverny 1953-1963", conduzida por pesquisadores do CERFI (Centre d'études, de recherches et deformation institnelles), fornece um relato impressionante sobre a complexidade envolvida no processo de feitio da grade. Veja "Histoires de La Borde"
} 


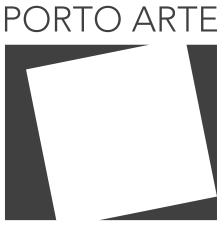

Revista de Artes Visuais

$\vee 25 n .44$

$\mathrm{Jul} / \mathrm{dez} 2020$ e-ISSN: 2179-8001

Guattari sugere ainda que associado à grade está o surgimento do que ele descreve como uma linguagem vibrante, uma linguagem que permitiria a expressão dos problemas da instituição. Como Guattari afirma: "[Seu] sistema está conectado com a invenção de uma linguagem, com seu modo particular de nomear diferentes tarefas e com uma retórica particular, e essa é a única maneira de tratar certos problemas" (1998, p. 12-13). O fato de a linguagem ter desempenhado um papel crucial no coletivo institucional só faz sentido se entendermos como ela serviu mais do que uma mera função tecnocrática. Ao se entregar à discussão do cotidiano, a linguagem revelou, em um gesto pragmático, seus territórios de conflito e produção. Isso acontece quando a afirmação se referiu a mais do que a si mesma, quando revelou o como, o onde e o porquê - ou, em outras palavras, sua política.

Nesse sentido, existem de fato referências ao surgimento do que é descrito como uma "língua local" em La Borde (Guattari, 1995, p.9), desenvolvendo-se a partir do intercâmbio de tarefas materiais e sociais, conhecimentos técnicos e especializados, um aprendizado coletivo de psicopatologia. Por exemplo, o uso de termos psiquiátricos foi ajustado e revisado de acordo com o uso local e o aprendizado coletivo de psicopatologia. Havia também o jargão de La Borde (a S.C.A.J. ou Souscommission d'Animation de la Journée e o B.C.M. ou Bureau de Coordination Médicale são exemplos disso). Isso contrasta com a adoção de semiologias gerais e manuais de diagnóstico como o $\mathrm{DSM}^{6}$ que exemplificam o que poderia ser descrito como uma axiomatização semiológica ${ }^{7}$ da multiplicidade do mental e cuja utilidade dificilmente se justifica em termos terapêuticos. Porque, como afirmei antes, os sucessos dessa forma específica de "invenção de uma linguagem" (Guattari, 1998, p. 12-13), como Guattari a chama, têm pouco a ver com a própria linguagem. Em vez disso, têm a ver com o fato de que este é um modo particular de semiotização que é de natureza coletiva e que retorna a linguagem às condições de sua existência, antes de tudo. A linguagem é aberta à disputa como parte de uma situação existencial concreta que a força a lidar com uma pragmática mais ampla que a excede.

Nesse sentido, o abandono da figura do especialista em análise institucional é justamente um esforço para estimular uma produção de semiotização em relação aos problemas imediatos e concretos. 0 crucial não foi a invenção de uma linguagem, mas a criação de fóruns de semiotização, como criações coletivas que poderiam influenciar na transformação da própria instituição. Em última análise, é importante chamar a atenção para a articulação entre a discursividade coletiva e o processo organizacional - ou seja, como a revisão constante da grade retroalimentaria a própria instituição.

\footnotetext{
6- American Psychiatric Association, Diagnostic and Statistical Manual of Mental Disorders (DSM), Washington, Dc: American Psychiatric Association, 1952 (first edition). O DSM está atualmente em sua quinta edição, publicada em 2013

7- Este foi o argumento da minha apresentação no seminário Axiomatic Earth, intitulado "The Financialization of the Mental" ("A Financialização do Mental”) durante o "Technosphere issue, Anthropocene Curriculum and Campus, House of World Cultures (HKW), Berlim, 2016.
} 


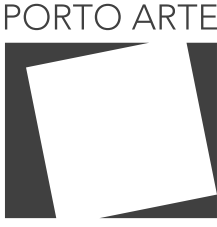

Revista de Artes Visuais

v. 25 n.44 Jul/dez 2020 e-ISSN: 2179-8001

Figura 4. Cozinha em La Borde. Início dos anos 70. Direitos de autor: Clínica La Borde.

\section{A grade como instituição}

De fato, a grade era um protocolo organizacional formal que fazia uso de um mecanismo de quantificação muito básico e pobre. 0 método em si era mínimo. Como Guattari afirmou: "[A] grade emprega tempo inscrito em um pedaço de papel" (Guattari, 1977, p. 271). E, no entanto, era um meio de criar coletivamente uma instituição. Em vez de impor um congelamento do ethos criativo da instituição, a grade foi uma formalização expressiva dessa agência coletiva no tempo. Por sua vez, essa formalização agiu sobre a matéria institucional - ou seja, transformando-a em um pressuposto recíproco.

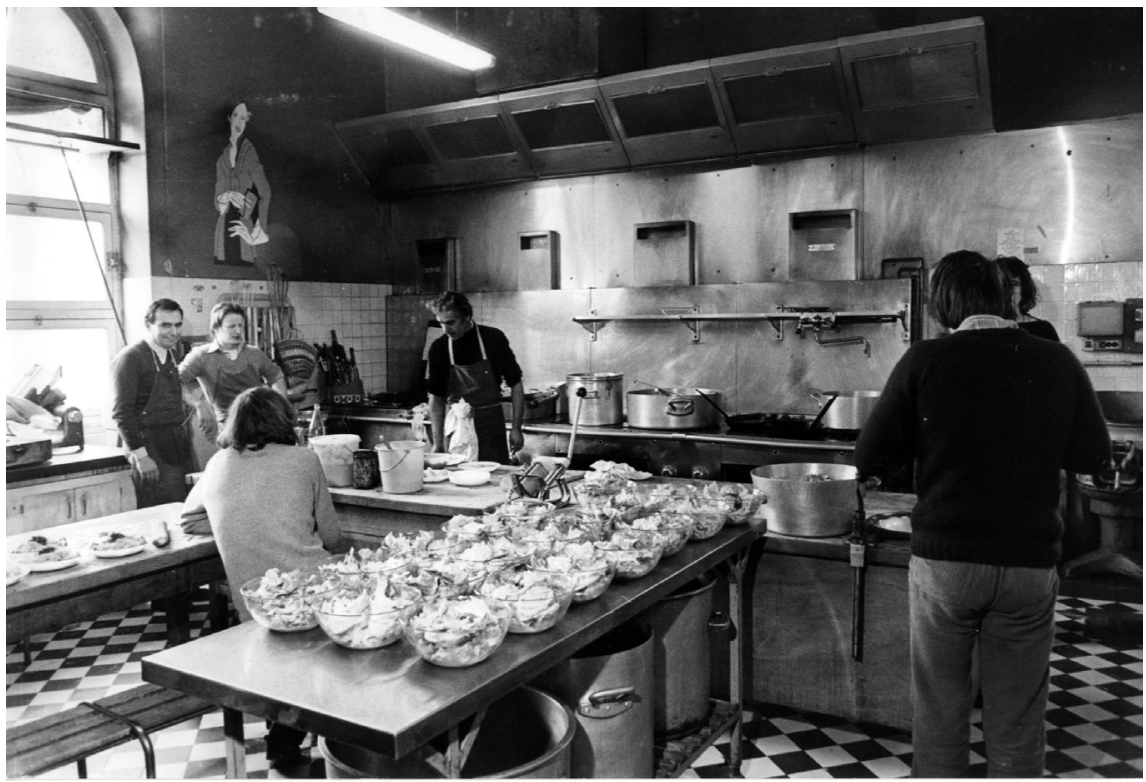

É aí que entra uma função de semiotização coletiva - que pode ser descrita como uma práxis semiótica de grupos e indivíduos; uma que serve não a uma axiomatização geral dos problemas da existência, mas a uma formalização não redutiva da vida coletiva. Para que isso aconteça, é fundamental que o significado extraído do sistema de quantificação da grade seja de responsabilidade do fórum coletivo que primeiro a montou. Indo um pouco além, essa capacidade de autoguiar os processos de semiotização é vital para a autonomia do coletivo institucional. Isso se encaixa na descrição de Guattari da semiótica a-significante, que prescreve um tipo de relação entre signos e coisas, pela qual o semiótico e o material se conectam diagramaticamente, envolvendo uma produção existencial do referente. Veja o exemplo da física teórica e da notação musical. Aqui, os signos fazem parte da produção material e se contrapõem a redundâncias semiológicas que representam e oferecem "equivalentes" de realidades. Nesse sentido, a grade pode ser entendida como um protocolo essencialmente a-significante. 


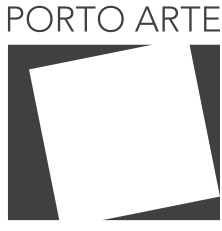

Revista de Artes Visuais

$\vee 25 n .44$ $\mathrm{Jul} / \mathrm{dez} 2020$ e-ISSN: 2179-8001

Assim, em vez de lançar o pessimismo nos processos institucionais ${ }^{8}$, Guattari e Oury propuseram uma reformulação inestimável das práticas sociais e institucionais. "Uma discussão sobre o processo de institucionalização não tem nada a ver com organogramas e regulamentos pré-estabelecidos", escreve Guattari: "mas sim com as possibilidades de mudança inerentes às trajetórias coletivas - atitudes evolutivas, auto-organização, e a assunção de responsabilidades" (Guattari e Rolnik, 1986, p. 255).

Tal compreensão dos mecanismos formais e processos institucionais que afirmam a possibilidade de sua não redutividade e de seu potencial constitutivo (aquele que não subtrai a multiplicidade do coletivo de sua formalização) ainda é extremamente relevante hoje. Muito tem sido escrito sobre os mecanismos de axiomatização e semiotização redutiva. Porém, pouco se disse sobre como os processos de formalização - que não são um fim em si mesmos - poderiam ser usados para informar os processos institucionais coletivos que não são redutivos por natureza e, portanto, poderiam inaugurar e marcar mudanças sociais e políticas importantes.

\section{REFERÊNCIAS}

Deleuze, Gilles. e Guattari, Félix. O Anti-Édipo: Capitalismo e esquizofrenia 1, tr. de Joana Moraes Varela e Manuel Maria Carrilho. Lisboa: Assírio \& Alvim, 1996 [1972].

Goffey, Andrew. "Guattari and Transversality: Institutions, analysis and experimentation," Em: Radical Philosophy, vol. 195 (2016): p. 38-47.

Guattari, Félix. "La 'Grille', Chimères, vol. 34, Autumn (1998): Disponível em: <https:// www.revue-chimeres.fr/Chimeres-no-34-La-grille>.

Guattari, Félix. La Révolution Moléculaire. Fontenau-sous-Bois: Éditions Recherches, 1977, p. 271

Guattari, Félix. "La Borde: A clinic unlike any other," Em: Sylvère Lotringer (ed.), Chaosophy. Los Angeles: Semiotext(e), 1995 [1977], p. 176.

Guattari, Félix. e Rolnik, Suely, Micropolítica: Cartografias do Desejo. Petrópolis: Editora Vozes, 1986. 


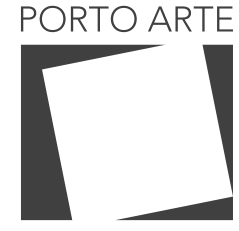

Revista de Artes Visuais

v. $25 n .44$

Jul/dez 2020 e-ISSN: 2179-8001

Texto submetido em: 18/11/2020 Texto publicado em: 19/12/2020

\section{Susana Caló}

Investigadora em filosofia e psicopolítica. Nos últimos dez anos tem investigado e publicado sobre o movimento da psicoterapia institucional, dedicando-se também à reconstrução de histórias menores do pós-guerra da psiquiatria mapeando as suas intersecções com lutas ativistas e sócio-políticas mais amplas. Escreve neste momento, com Godofredo Pereira, um livro sobre a cooperativa de investigação fundada por Félix Guattari, intitulado: CERFI: Programação Institucional, Equipamento Colectivo e Análise Militante. É investigadora no King's College London e membro dos colectivos Transversal Collective, Other Ways to Care e Network for Institutional Analysis. 